\title{
PRELIMINARY STUDY ON EPIPHYTIC MICROALGAE ON AQUATIC PLANTS AT SOHAG DISTRICT.
}

\author{
Zeinab A. Ahmed \\ Botany Department, Faculty of Science, Sohag University.
}

\begin{abstract}
This Study investigates epiphytic algal flora on aquatic plants collected from different sites in the River Nile and irrigation canals at Sohag district, Egypt during June 2007. At the same time, the physico - chemical properties of water samples were determined. A total of 105 species related to forty eight genera of micro-algae were identified as epiphytic communities on different aquatic plant (Eichhornia crassipes, ceratophyllum demersum, Myriophyllum spicatum, phragmites australis). Of which sixty one species related to twenty five genera belonging to Bacillariophyta, thirty species of twelve genera belonging to Chlorophyta. Ten species related to eight genera belonging to Cyanophyta, one species belonging to Xanthophyta and three species of three genera belonging to Euglenophyta.
\end{abstract}

Key words: Epiphytes-Microalgae-Aquatic plants.

\section{Introduction}

Epiphytic micro-algae are important common constituents of the autotrophic community of a macrophyte-oriented aquatic ecosystem. Epiphyton are primary producers that fix carbon and uptake essential nutrients from the water column, thereby making these nutrients accessible at higher trophic levels. These autotrophs are often overlooked due to their minute size; however, recent work has demonstrated their importance in terms of productivity (Burkholder and Wetzel, 1990), biomass (Zimba, 1995) and as a food source for higher trophic levels (Kitting et al., 1984; Sullivan and Moncreiff, 1990). Efficient removal of epiphyton from host tissues must be accomplished to assess the importance of macrophytes and epiphytes in ecological studies (Wetzel, 1983; Sand-Jensen $\boldsymbol{e t}$ al., 1989; Goldsboroy and Hickman, 1991). Epiphytic communities on Thalassia testudinum from Grand Cayman, British West Indies: Their composition, structure, and contribution to lagoonal sediment have been studied 
by Corlett and Jones (2007). El-Shahed and Fathy (2000) studied the diatom assemblages associating Cladophora glomerata Kutz in Egyptian fluvial environment. Burkholder et al. (1990) and Cattaneo and Kalff (1978) identified two distinct components of the epiphytic flora: the loosely attached and tightly attached or admate component, these two components exhibit different physiological activities.

Preliminary investigations were carried out on the epiphytic algae, revealed list of the species and frequency of some dominant taxa in Turkish fresh waters (Yildiz, 1987; Şen and Aksakal, 1988; Obali et al., 1989; Dere et al., 2002; Saunders et al., 2003).

The present paper presents the characteristics of epiphytic micro algae accompanying aquatic plants in the river Nile, the drainage canal and waste water canal.

\section{Methods and Materials}

\section{Sampling locations:}

Aquatic plants were collected during June 2007 from four sites.

Site I: represents industrial pollution site. It is located at the main stream of the River Nile east bank (Fig. 1) near the outlet of Pepsi Cola factory. (N: 26 33' 29"; E: $31^{\circ} 42^{\prime} 33^{\prime \prime}$, depth is $1-2 \mathrm{~m}$ approximately).



Figure (1): Map of the study area. 
Site II: represents agricultural waste water drainage site, (Fig. 1) located at Sohag Al-Gharby drainage canal (N: $26^{\circ} 32^{\prime} 18^{\prime \prime}$; E: $31^{\circ} 41^{\prime} 79^{\prime \prime}$, depth is $1-1.5 \mathrm{~m}$ approximately).

Site III: was located opposite to the major outfall of domestic wastewater

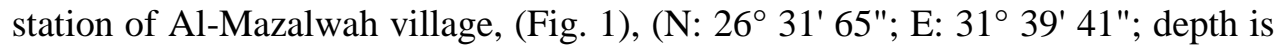
0.5-1 $\mathrm{m}$ approximately).

Site IV: represents a mixture of domestic and agricultural wastewater site (Fig. 1). It is located near Rawafeih Al-Kusair village (N 26 31' 16"; E $31^{\circ} 42^{\prime}$ 39"; depth is $0.5-1.25 \mathrm{~m}$ approximately). Echhornia crassippes were collected from site ( I, II, IV); Ceratophyllum demersum were collected from site (I and III). Myriophyllum spicatum from site (I) and Phragmites from site (III).

\section{Sampling processing:}

\section{Algal identification and photography.}

Macrophytic plants were harvested by carefully removing individuals from substrate and putted in plastic bags in a darkened cooler. Processing of plant samples never exceeded $4 \mathrm{hrs}$ from collection time. In the laboratory a definite weights of Eichhornia, Ceratophyllum, Myriophyllum and Phragmites were placed in plastic bottles. Deionized water $(100 \mathrm{ml})$ was used to rinse each plant, and then the bottle was capped and manually shaken for 10 second. Host plant materials was removed from the epiphytic slurry and placed in second bottles. Deionized water $(100 \mathrm{ml})$ was again added and the bottle shaken for an additional 10 seconds. Host plant was removed and the first and the second epiphyte slurry were collected. Lugl's iodine solution was added for preservation and then the volume $(200 \mathrm{ml})$ was concentrated to $(20 \mathrm{ml})$ for taxonomic enumeration. For taxonomic identification, microscope Olympus Model BX51+R F, with digital camera Olympus D P12-2 was used.

Identification of the algal species was carried out according to Bourrelly (1968, 1970; Prescott (1978) and Cox (1996).

\section{Physical parameters:}

Temperature and $\mathrm{pH}$ were measured in situ using thermometer and $\mathrm{pH}$ meter (HANNA model 211).

\section{Chemical analysis:}

Different methods of chemical analysis were performed for water samples. Dissolved oxygen using oxygen meter (GLX. PASCO. PS-2002); conductivity using conductivity meter (MARTINI. Mi 170 Bench Meter) ; total organic matter, total soluble salt, chloride according to (American Public Health Association, 1981), Bicarbonate according to (Jackson, 1977), soluble 
phosphorus according to (Woods and Mellon, 1941), nitrite described by (Strickland and Parasons, 1965), nitrate according to (Deutsche Einheitsverfahren Zur Wasser-Abwasser and Schlammuntersuchung, 1960) and ammonia described by (Dewis and Freitas, 1970) were measured. Cations like $\mathrm{Ca}^{+2}$ and $\mathrm{Mg}^{+2}$ according to (Schwarzenback, 1948) were also measured.

\section{Results and Discussion}

At the sampling stations during June, 2007, the average physical and chemical parameters were measured: water temperature (range $27-29{ }^{\circ} \mathrm{C}$ ), $\mathrm{pH}$ (range 7.7-8.4), conductivity (range 520-900), and the highest value were found in site IV and site III. Dissolved oxygen (range 6-12.3), the highest values were in site IV and site I.

The values of total soluble salts, (range170-450 ppm) the highest values were in site III, IV, the highest values of total organic matters; nitrate, nitrite and the soluble phosphorus were found in site III, IV. In addition, the highest value of $\mathrm{Ca}^{+2}$ and $\mathrm{Mg}^{+2}$ were found in site I, IV (Table 1).

Table (1): physical and chemical analysis of water samples from 4 sites.

\begin{tabular}{|c|c|c|c|c|}
\hline \multirow{2}{*}{ Physical and chemical analysis } & \multicolumn{4}{|c|}{ Water sites } \\
\hline & Site (I) & Site (II) & Site (III) & Site (IV) \\
\hline Water temperature & $27^{\circ} \mathrm{C}$ & $27^{\circ} \mathrm{C}$ & $29^{\circ} \mathrm{C}$ & $27^{\circ} \mathrm{C}$ \\
\hline pH value & 7.65 & 8.20 & 7.9 & 8.38 \\
\hline Dissolved Oxygen ( mg/L) & 12.30 & 8.00 & 6.00 & 10.80 \\
\hline Conductivity) $\mu$ mohs $\mathrm{cm}^{-1}$ & 520 & 340 & 900 & 885 \\
\hline Total soluble salts ( $\mathrm{mg} / \mathrm{L}$ ) & 259 & 170 & 450 & 442 \\
\hline$\% \mathrm{Na} \mathrm{Cl}$ & 1.0 & 0.7 & 1.8 & 1.7 \\
\hline Total organic matters & 0.501 & 0.931 & 0.960 & 1.000 \\
\hline $\mathrm{Cl}^{-}(\mathrm{mg} / \mathrm{L})$ & 0.006 & 0.0035 & 0.014 & 0.007 \\
\hline $\mathrm{HCO}_{3}(\mathrm{mg} / \mathrm{L})$ & 0.61 & 0.61 & 0.61 & 0.915 \\
\hline $\mathrm{NO}_{3}^{-}(\mu \mathrm{g} / \mathrm{L})$ & 47.60 & 38.36 & 49.84 & 75.60 \\
\hline $\mathrm{NO}_{2}^{-}(\mathrm{mg} / \mathrm{L})$ & 0.12 & 0.12 & 0.24 & 1.08 \\
\hline Soluble phosphorus $\left(\mathrm{PO}_{4}^{--}\right)(\mu \mathrm{g} / \mathrm{L})$ & 6.59 & 1.24 & 12.90 & 8.33 \\
\hline $\mathrm{Ca}^{++}(\mathrm{mg} / \mathrm{L})$ & $\mathbf{3 0 . 0 0}$ & 14.00 & 19.00 & 26.00 \\
\hline $\mathrm{Mg}^{++}(\mathbf{m g} / \mathbf{L})$ & 42.00 & 19.20 & 40.20 & 44.40 \\
\hline
\end{tabular}

Micro-algae identified in the epiphytic slurry included a total of 105 species related to 48 genera as epiphytic communities on different aquatic plants (Eichhornia crassipes, Ceratophyllum demersum, Myriophyllum spicatum, Phragmites australis) Plate (1). The major taxonomic groups during the summer month (June, 2007) are shown in Table (2). 


\section{Plate (1)}

Figures (1 and 2): Photographs of Spirulina and diatom on Echhornia crassipes leaves.

Figures (3, 4 and 5): Protoderma viride.

Figure (6): Cocconies placentula on Myrophyllum spicatum stem. Figures (7 and 8): Epiphytes on Ceratophyllum demersum stem.

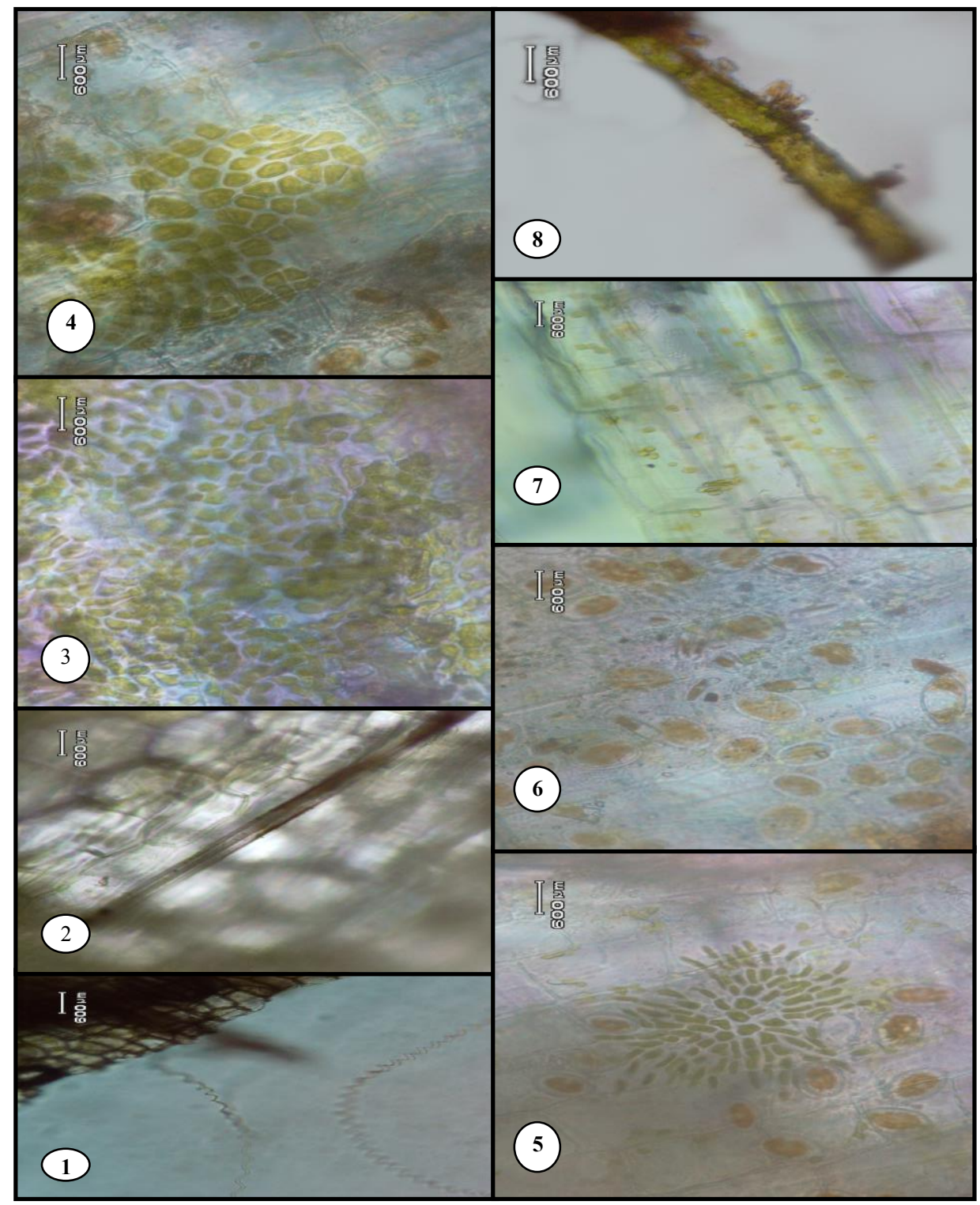


Table (2): Occurrence of algal taxa in the studied sites.

\begin{tabular}{|c|c|c|c|c|c|c|c|}
\hline \multirow[b]{2}{*}{ Algal species } & \multicolumn{4}{|c|}{$\begin{array}{c}\text { Site } \\
\text { I }\end{array}$} & \multirow{2}{*}{ 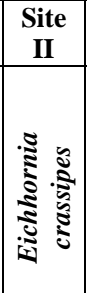 } & \multirow{2}{*}{ 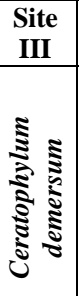 } & \multirow{2}{*}{ 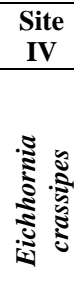 } \\
\hline & 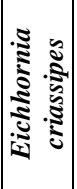 & 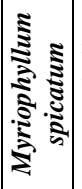 & 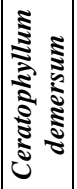 & 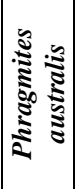 & & & \\
\hline \multicolumn{8}{|l|}{ Chlorophyta } \\
\hline 1-Chlamydocapsa planctonica fott & - & - & - & - & - & + & - \\
\hline 2- Closterium parvulum & - & - & - & - & - & + & - \\
\hline 3- Crucigenia rectangularis (A. Braun) & - & - & - & + & - & + & - \\
\hline 4- Cosmarium granatum Brebisson. & - & + & + & + & - & + & - \\
\hline $\begin{array}{l}\text { 5- Coelastrum cambricum Archer var. intermedium } \\
\text { Bohlin }\end{array}$ & - & - & + & + & - & + & - \\
\hline 6- Coelastrum microporum Naegli. & - & + & - & + & - & + & - \\
\hline 7- Coelastrum cambricum Archer. & - & + & - & + & - & + & - \\
\hline 8- Coelastrum reticulatum (Dang.) senn. & - & - & - & - & - & + & - \\
\hline 9- Pediastrum biradiatum var. emarginatum Prescott. & - & - & - & - & - & + & - \\
\hline 10- Pediastrum tetras Ralf. & - & + & + & + & - & + & - \\
\hline 11- Pediastrum sculptatum G. M. Smith. & - & - & - & - & - & + & - \\
\hline 12- Pediastrum simplex Meyen. & - & - & - & + & - & + & - \\
\hline 13-Pediastrum boryanum Meneghini. & - & - & + & - & - & + & - \\
\hline 14- Coleochaete orbicularis Pringsheim. & - & - & + & - & - & - & - \\
\hline 15- Scenedesmus bijuga (Turp.) Largerheim. & - & + & + & + & - & + & - \\
\hline 16- Acutodesmus acuminatus. & - & + & + & - & - & + & - \\
\hline 17-Scenedesmus opoliensi P. Richer. & - & + & - & - & - & + & - \\
\hline $\begin{array}{l}\text { 18- Scenedesmus armatus chodat var. Bicaudatus } \\
\text { (Chodat). }\end{array}$ & - & - & - & - & - & + & - \\
\hline 19- Scenedesmus arcuatus Lemmermann & - & - & + & - & - & - & - \\
\hline 20-Scenedesmus quadricauda (Turp.). & - & + & + & + & - & + & - \\
\hline 21- Scenedesmus denticulatus (Lagerheim.) & - & - & - & - & - & + & - \\
\hline 22- Spirogyra subsalsa & - & - & + & - & - & + & - \\
\hline 23-Ulothrix zonata & - & - & - & - & - & + & - \\
\hline 24- Oedogonium spirostriatum Tiffany. & - & - & + & - & - & + & - \\
\hline 25-O. pyriforme Wittrock & - & - & + & - & - & + & - \\
\hline 26-O.pringsheimii Cramer & - & - & + & - & - & + & - \\
\hline 27- Oedogonium inclusum Hirn. & - & - & + & - & - & + & - \\
\hline 28- Oedogonium.boheanicum (Hirn). & - & - & + & - & - & + & - \\
\hline 29- Oedogonium pisanum Wittrock. & - & - & + & - & - & + & - \\
\hline \multicolumn{8}{|l|}{ Cyanophyta } \\
\hline 30-Aphanizomenon.flosaqua. Ralf & - & - & - & - & - & + & - \\
\hline 31-Anabaena aequalis Borge. & - & - & - & - & - & + & - \\
\hline 32-Calothrix stigmalis. & - & - & + & - & - & - & - \\
\hline 33-Lyngbya limnetica. & - & - & + & - & - & + & - \\
\hline 34- Nostoc.sp. & - & - & - & - & - & + & - \\
\hline 35- Nodularia spumigena Mertens. - & - & - & + & - & - & - & - \\
\hline 36- Oscillatoria brevis var. neapolitiana. & - & - & + & - & - & + & - \\
\hline 37- O. articulata Gardner. & - & - & + & - & - & - & - \\
\hline 38-O. $s p$ & - & - & + & - & - & + & - \\
\hline
\end{tabular}




\begin{tabular}{|c|c|c|c|c|c|c|c|}
\hline 39- Spirolina laxissima. & + & - & + & - & - & - & - \\
\hline \multicolumn{8}{|l|}{ Bacillariophyta } \\
\hline 40- Amphora Montana krasske & - & - & + & - & - & + & - \\
\hline 41- Amphora ovalis Kutz & + & + & + & + & - & + & - \\
\hline 42- Amphora commutata Grunow & - & - & - & + & - & + & - \\
\hline 43- Amphora veneta kutz & - & - & + & + & - & + & - \\
\hline 44-Cocconies placentula Her. & + & + & + & + & + & + & + \\
\hline 45- Cyclotella meneghiniana Kutz & + & + & + & + & + & + & + \\
\hline 46- Cyclotella comta .Ehr. & - & + & + & - & - & + & - \\
\hline 47- Cyclotella kutzingiana Thwattes. & - & + & + & + & - & + & + \\
\hline 48-Cyclotella striata kutz. & - & + & + & - & - & + & - \\
\hline 49- Cyclotella operoulata Kutz. & - & + & + & + & - & + & + \\
\hline 50-Stephanodicus hantzschii Kutz. & - & - & + & - & - & + & - \\
\hline 51- Diplonies finnica (Ehr.) kirchener. & - & - & + & - & - & + & - \\
\hline 52- Cymbella affinis Kutz. & - & + & - & + & - & + & - \\
\hline 53- Encyonema caespitosa (kutz). & - & + & + & + & - & + & - \\
\hline 54- Cymbella cistula (Ehren)Kichner. & - & + & - & + & - & + & - \\
\hline 55- Cymbella tumida (Brebison.) van Heurch. & - & + & - & - & - & + & - \\
\hline 56- Cymbella proxima Remier. & - & - & - & - & - & + & - \\
\hline 57- Gomphonema gracile Ehrenberg. & - & - & + & - & - & + & - \\
\hline 58- Gomphonema augur Ehren. var turis Ehren. & - & - & - & - & - & + & - \\
\hline 59- Gomphonema augur Ehren. var. sphaerophorum. & - & - & - & + & - & + & - \\
\hline 60- Gomphonema augur Ehren. var. augur & - & - & + & + & - & + & - \\
\hline $\begin{array}{l}\text { 61- Gomphonema olivaceum (Hornemann.) var. } \\
\text { olivaceum. }\end{array}$ & - & + & + & + & - & + & - \\
\hline 62- Melosira granulate.Ralfs. & + & + & + & + & + & + & + \\
\hline $\begin{array}{l}\text { 63- Fragillaria .construnes Grunow var.venter } \\
\text { Grunow. }\end{array}$ & - & - & + & - & - & + & - \\
\hline 64- F. producta var. acuta.(E.e.p.) A.Ca & - & - & - & - & - & + & - \\
\hline 65- F. capucina (Desmazieres). & + & + & + & + & + & + & + \\
\hline 66- $F$. crotunensis var.oregona. & - & - & + & + & - & + & - \\
\hline 67- Nitzschia frustuluns var perpusila (rabh.). & - & - & + & - & - & + & - \\
\hline 68- $N$. amphiboides Hust. & - & - & - & - & - & + & - \\
\hline 69- N.elegans. & - & - & + & - & - & + & - \\
\hline 70- N. fonticola var. genuina A.Cl. & - & - & - & - & - & + & - \\
\hline 71- N. sigmoidea. & - & + & + & + & + & + & - \\
\hline 72-N. amphibia var.acutiuscula Grun. & - & - & - & - & - & + & - \\
\hline 73-N. palae. & - & - & - & - & - & + & - \\
\hline 74-Navicula capitatoradiosa Gernain. & - & - & + & - & - & + & - \\
\hline 75- N.viridula.Kutz.Ehren.var.viridul & - & - & + & - & - & + & - \\
\hline 76- N.menisculus Schumnn. & - & - & - & - & - & + & - \\
\hline 77- N. exigua .Husdt. & - & - & - & - & - & + & - \\
\hline 78- N.gastrum (Ehren.) Kutz.var.gastrum. & - & - & - & - & - & + & - \\
\hline 79- N. peregrine (Ehrin.) Kutz. & - & - & - & - & - & + & - \\
\hline 80-N.sp & - & - & - & - & - & + & - \\
\hline 81- Achnanthes minutissima Kutz.Grun. & - & - & + & - & - & + & - \\
\hline 82-Plerusigma angulatum (Quekett) W.Smith & - & - & - & - & - & + & - \\
\hline 83-Pinnularia brandelii Cleve. & - & - & - & - & - & + & - \\
\hline 84-P. acrosphaeria Rabenhorst. & - & - & + & - & - & + & - \\
\hline 85- P. nobilis (Ehren.) & - & - & + & - & - & + & - \\
\hline 86 - P. macilenta var. opulanta A.Ca. & - & - & - & - & - & + & - \\
\hline 87- Calonies silicula (Ehren) Cleve. & - & - & + & - & - & + & - \\
\hline 89 - Synedra ulna Ehr. & + & + & + & + & + & + & + \\
\hline 90- S.acus (kutz.) Hust. & + & + & + & + & + & + & + \\
\hline 91 - S. acus.var.angustissima Grunow. & + & - & - & - & - & - & - \\
\hline 92- S.crystallina (lyng.)Kutz. & - & - & - & + & - & + & - \\
\hline
\end{tabular}




\begin{tabular}{|c|c|c|c|c|c|c|c|}
\hline 93-Surirella gessneri & - & - & - & - & - & + & - \\
\hline 94- Stauronies anceps Ehren. & - & + & + & + & - & + & - \\
\hline 95- Tabbellaria venticosa & - & - & - & - & - & + & - \\
\hline 96- Bacillaria paradoxa Gmelin & + & - & + & - & - & - & + \\
\hline 97-G.truncatum Ehren & - & + & + & + & - & + & - \\
\hline 98-Gomphonema acuminatum.Ehr. & - & - & + & + & - & + & - \\
\hline 99- Melosira granulata Ralf var. angustissima & + & - & + & - & + & + & - \\
\hline 100- Synedra Capitata Ehr. & + & + & + & + & + & + & + \\
\hline 101- Calonies amphisbaena var. genuina. A. Ct. & - & + & - & - & - & - & - \\
\hline \multicolumn{8}{|l|}{ Xanthophyta } \\
\hline 102-Tribonema bombycinum (Ag.)Derbes and Solier. & - & - & + & + & - & + & - \\
\hline \multicolumn{8}{|l|}{ Euglenophyta } \\
\hline 103-Phacus sp. & - & - & - & - & - & + & - \\
\hline 104-Trachelomonas sp. & - & - & - & - & - & + & - \\
\hline 105-Euglena sp. & - & - & - & - & - & + & - \\
\hline Number of taxa/ site & 12 & 29 & 60 & 34 & 9 & 96 & 9 \\
\hline
\end{tabular}

Diatoms were the dominant group with 61 taxa related to 20 genera in the epiphytic community (Plates 2-5). Chlorophyta and Cyanophyta were the other main groups in the epiphytic flora 29 species of 9 genera belonging to Chlorophyta (Plate 6). Whereas, 10 species of 8 genera belonging to Cyanophyta (plate 7). Euglenophytes and Chrysophytes were the small group, 3 species of 3 genera belonging to Euglenophyta and one species belonging to Xanthophyta. This is in accordance with (Dere et al., 2002; Albay and Aykulu, 2002).

It was observed that loosly attached species of diatoms (primarily Cocconies placentula, Cyclotela meneghniana, Fragillaria capucina, Melosira granulata and Synedra ulna) were widespread in the flora of epiphytic diatoms found on all types of examined aquatic plants in all habitats. It has been recorded that high amount of filamentous and Chlorococcales Chlorophytes (Oedogonium spp., Spirogyra spp., Ulothrix sp., Scenedesmus spp., Pediastrum spp. and Coelastrum spp.) were found on Ceratophyllum demersum in site (I and III) and at site I Myriophyllum spicatum and phragmites have the same species except the filamentous types. This is in accordance with Albay and Aykulu (2002). Similar results were found by Muller (1996) who recorded high numbers of filamentous chlorophytes (Oedogonium spp., Spirogyra spp. and Mougeotia spp.) in shallow German lake. Cyanophyta represented by (Aphanizomenon flosaqua, Calothrix stagmalis, Nostoc sp., Anabaena aequalis, Lyngbya limnetica, Nodularia spumigena, Oscillatoria articulate and Oscillatoria brevis) were found on Ceratophyllum at site (I and III). Euglenophyta (Euglena sp., Phacus sp. and Trachelomonas sp.), in addition to Xanthophyta (Tribonema bombycinum) were found on Ceratophyllum demersum in site (III) (Table 2) where received nutriet rich water from sewage waters (Table1). At site (I and III) diatoms species (Gomphonema gracile, Gomphonema olivacium, Gomphonema augur, Gomphonema truncatum, Diplonies finnica, Cymbella affinis, Cyclotella striata, Navicula viridula, Nitzschia sigmoidea, Nitzschia amphibia, Pinnularia nobilis 
Plate (2): Bacillariophyta (I)

Figures: 1-Amphora Montana; 2-Amphora sp.; 3-A. commutate; 4-A. veneta; 5,6- Cocconies placentula; 7- Cyclotella meneghiniana; 8-C .comta; 9-C. kutzingiana; 10- C. striata; 11- C. operoulata; 12-Stephanodicus hantzschianus; 13,14-Diplonies finnica; 15-Cymbella affinis; 16-Encyonema caespitosum.

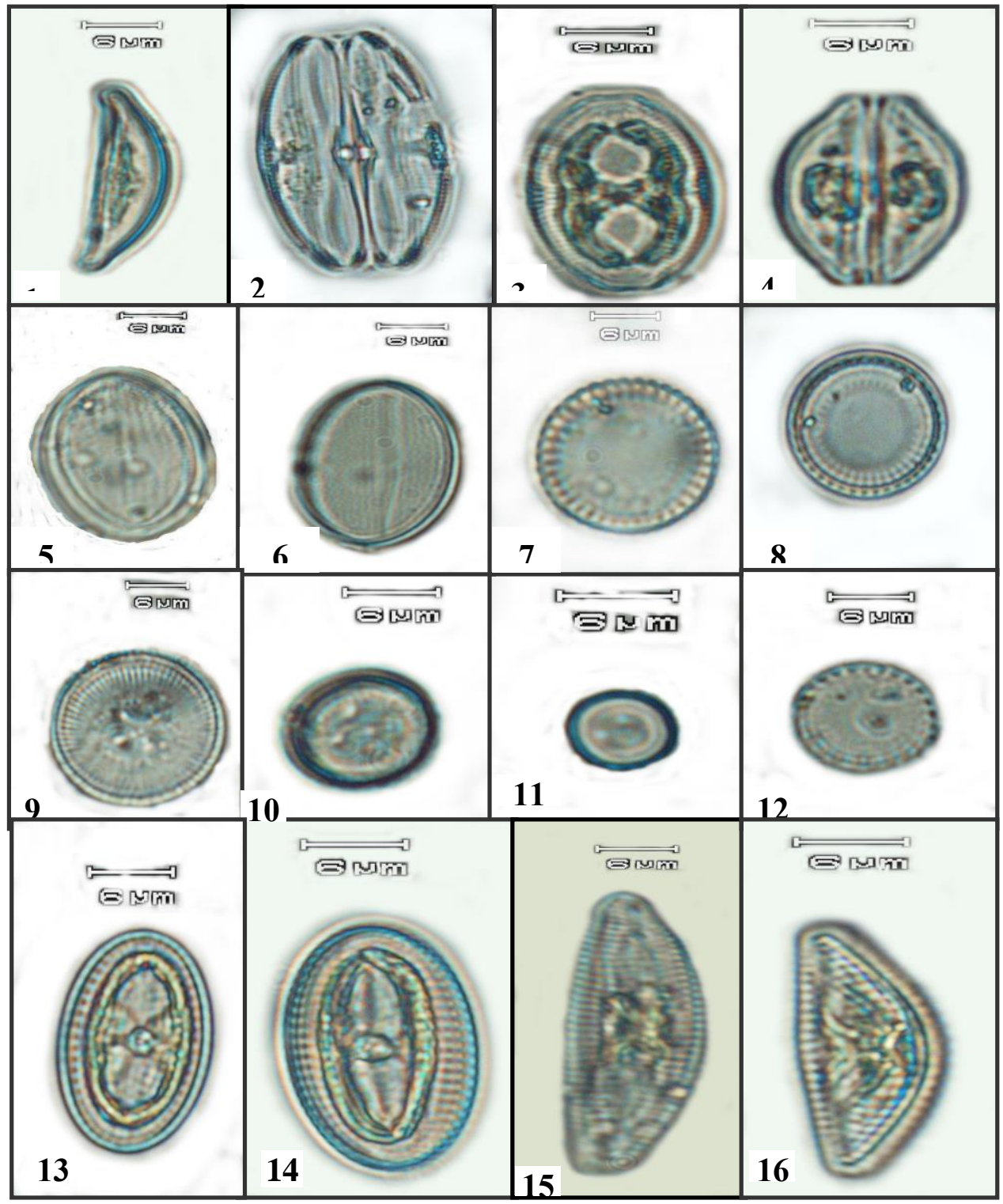


Plate (3): Bacillariophyta (II)

Figures:17,18,19,20-Cymbella cistula; 21-C. tumida; 22-C. proxima; 23-Gomphonema gracile; 24,25-G. augur Ehren. var turris; 26-G. augur var. Sphaerophorum; 27-G. augur var. Augur; 28-G.truncatum; 29-G.ventricosum; 30-G. olivaceum var olivaceum. olivaceuma

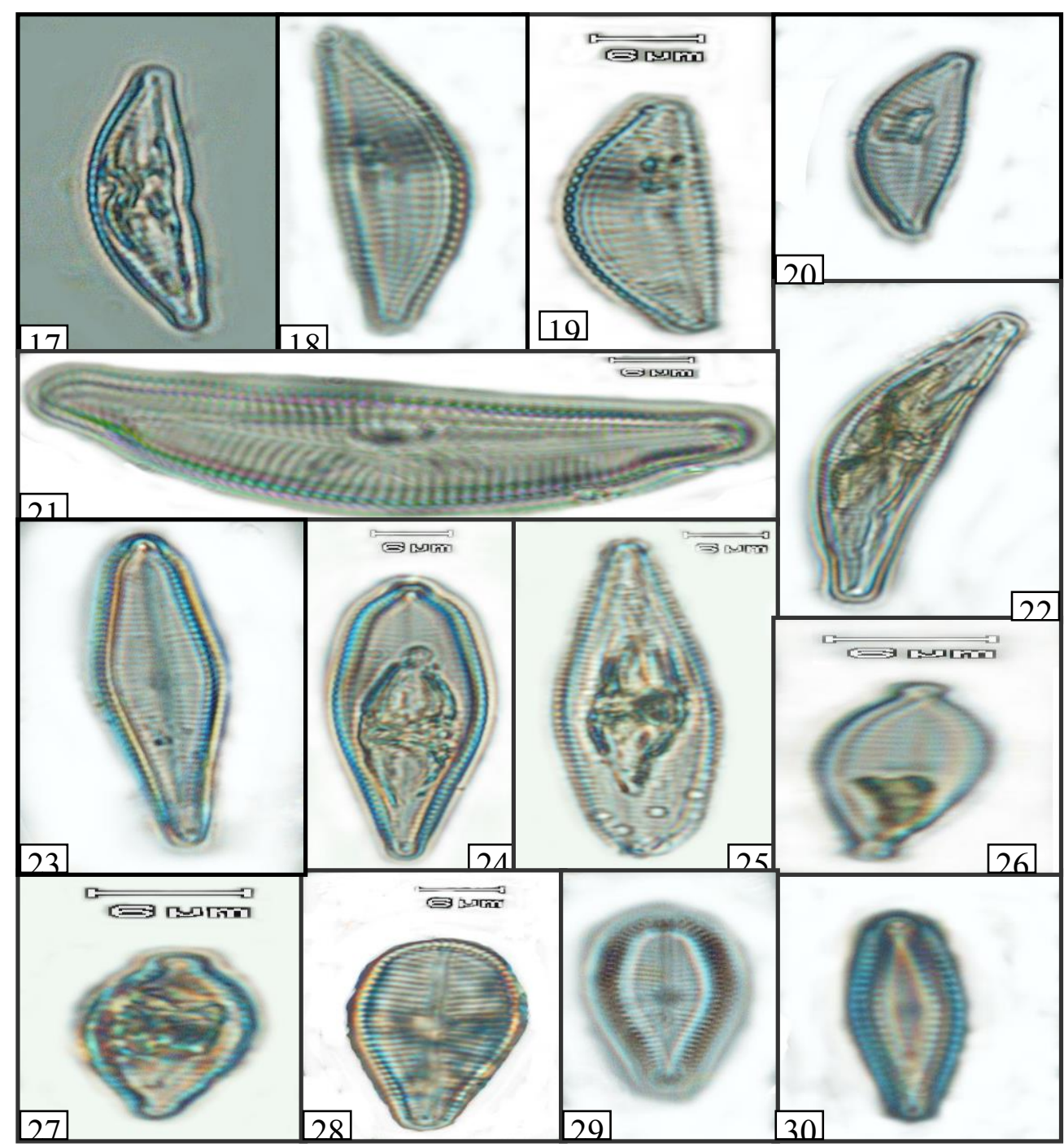




\section{Plate (4): Bacillariophyta (III)}

Figures: 31-Melosira granulate; 32-Fragillaria construnes var. venter; 33-F. producta var. acuta; 34-F. capunica; 35-F. crotunensis var. oregona; 36-Nitzschia frustuluns var perpusila; 37-N. amphiboides; 38 -N. elegans; 39-N. fonticola; 40-N. sigmoidea; 41-N. amphibia var. acutiuscula; 42-N. palae; 43- Navicula capitatoradiosa; 44-N. viridula var.viridula; 45-N. Menisculus; 46-N. Exigua, 47-N. gastrum var.gastrum;

48- $N$. peregrina; 49-N. sp.; 50, 51-Achnanthes minutissima.

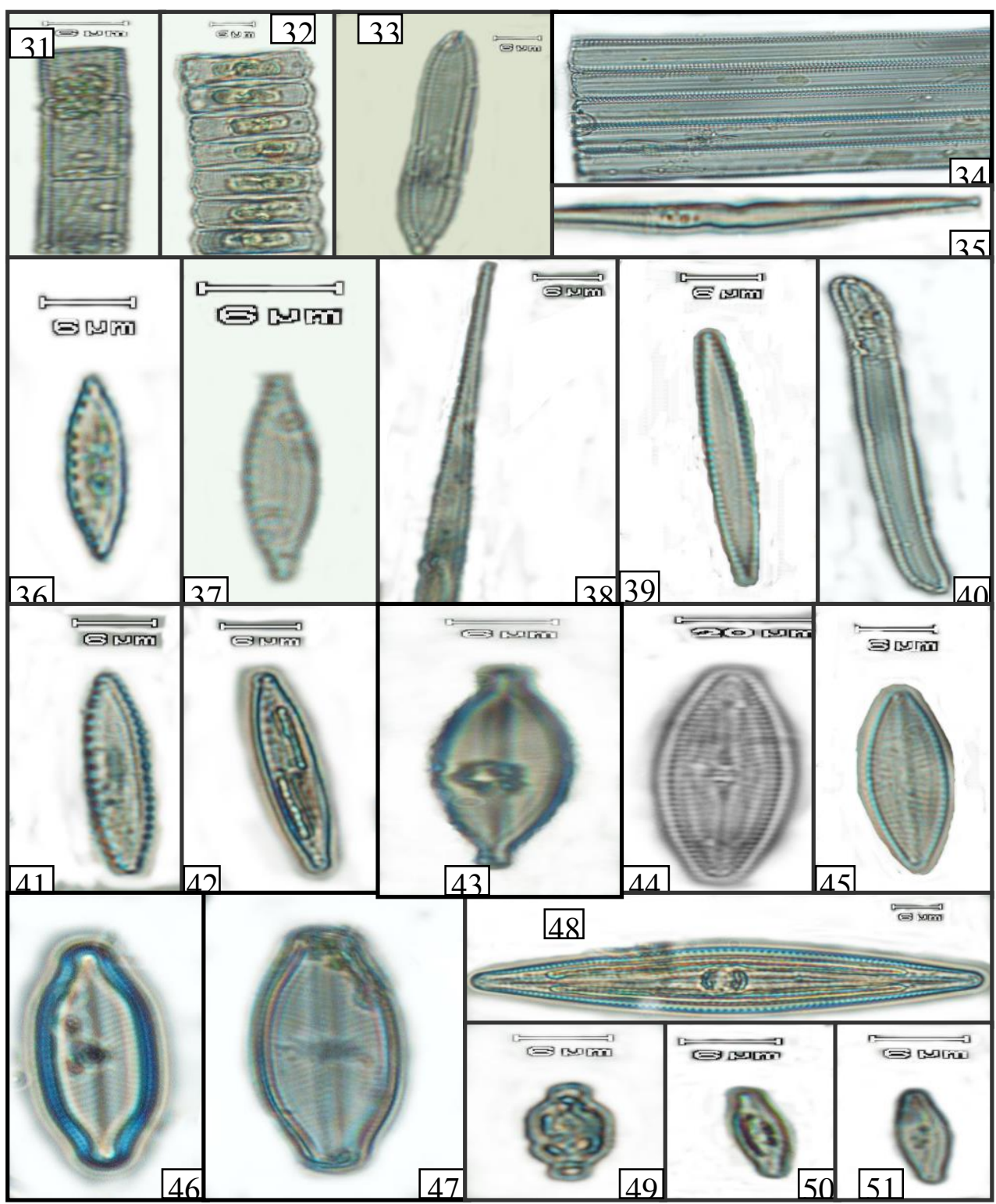




\section{Plate (5): Bacillariophyta (IV)}

Figures: 52-Plerusigma angulatum; 53-Pinnularia brandelii; 54-P. acrosphaeria; 55-P. nobilis; 56 -P. macilenta var. opulanta; 57-Calonies silicula; 58-Synedra ulna; 59-S. acus var. Radians; 60-S. acus var. angustissima; 61-S. crystallina; 62-Surirella gessneri; 63-Stauronies anceps; 64- Tabbellaria venticosa.

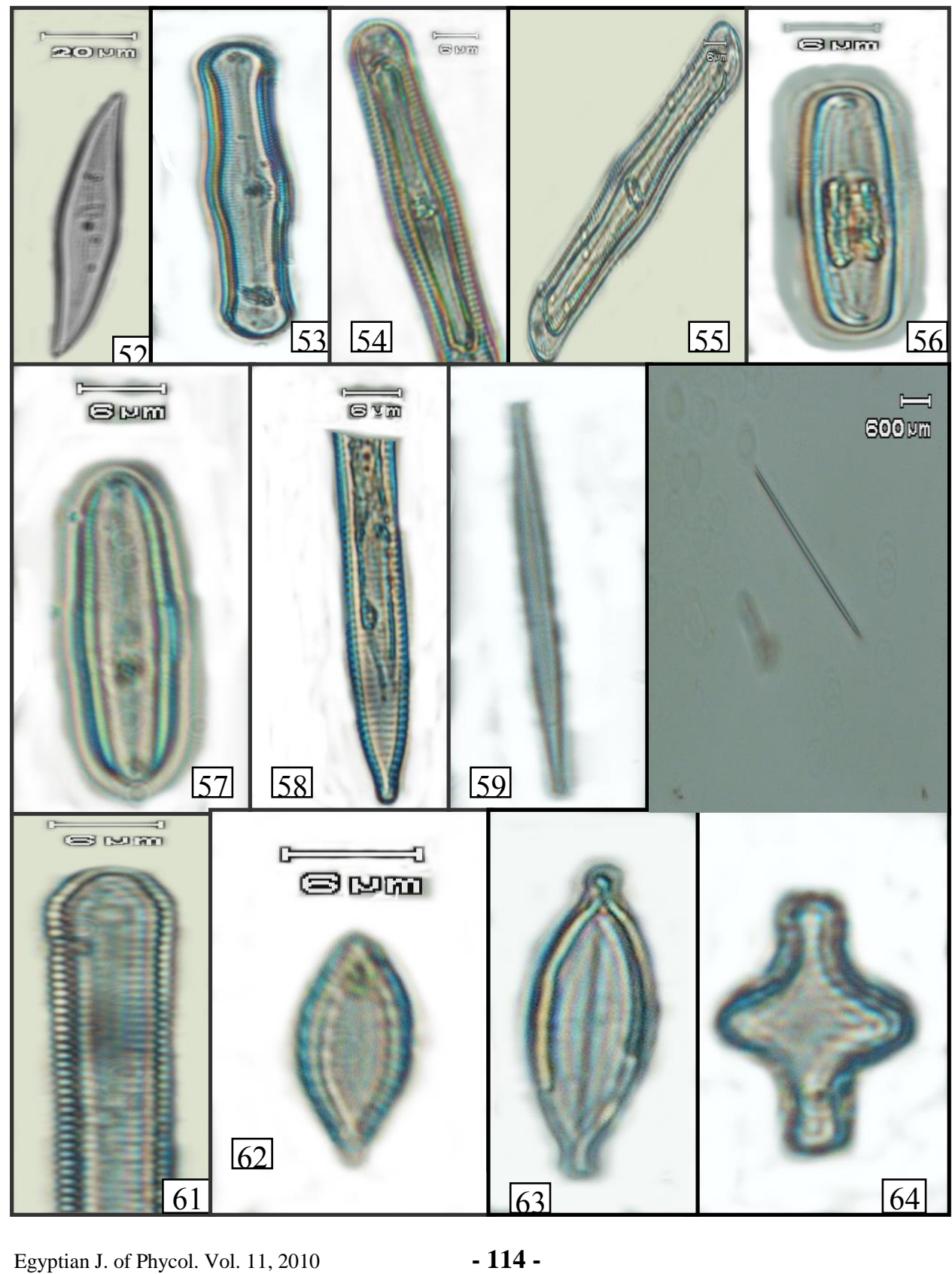




\section{Plate (6): Chlorophyta}

Figures: 1-Chlamydocapsa planctonica; 2-Closterium parvulum; 3-Crucigenia rectangularis; 4-Cosmarium granatum; 5-Coelastrum cambricum Archer var. Intermedium; 6-C. Microporum; 7-C. Microporum; 8-C. Retleulatum; 9-Pediastrum biradiatum var. emarginatum; 10-P. tetras; 11-P. sculptatum; 12-P. simplex; 13-P. boryanum; 14-Coleochaete orbicularis; 15-Acutodesmus acuminatus; 16-Scenedesmus bijuga; 17-S . opoliensis; 18-S. armatus chodat var. bicaudatus; 19-S. arcuatus; 20-S. quadricauda; 21-S. denticulatus; 22-Spirogyra sp.; 23-Ulothrix zonzta; 24-Oedogonium spirostriatum; 25-O. pyriforme; 26-O. pringsheimii; 27-O. inclusum; 28-

O.boheanicum; 29-O. Pisanum.

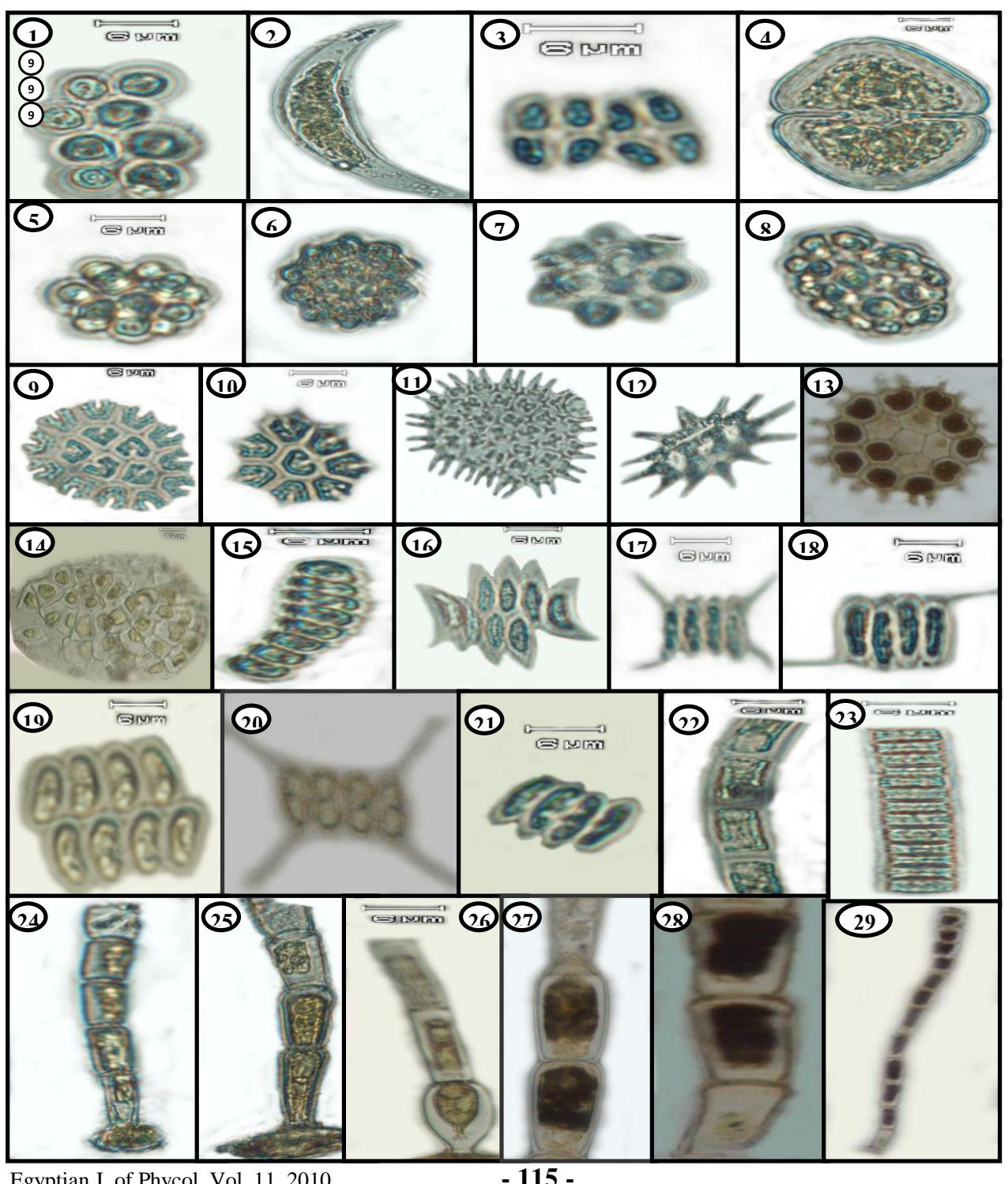




\section{Plate (7): Cyanobacteria}

Figures: 1- Aphanizomenon flosaqua; 2- Anabaena aequalis; 3-Calothrix stagmalis; 4-Lyngbia limnetica; 5- Nostoc sp.; 6- Nodularia spumigena; 7-Oscillatoria brevis var. neapolitana; 8-O. articulata; 9- O. sp.; 10-Spirulina laxissima.

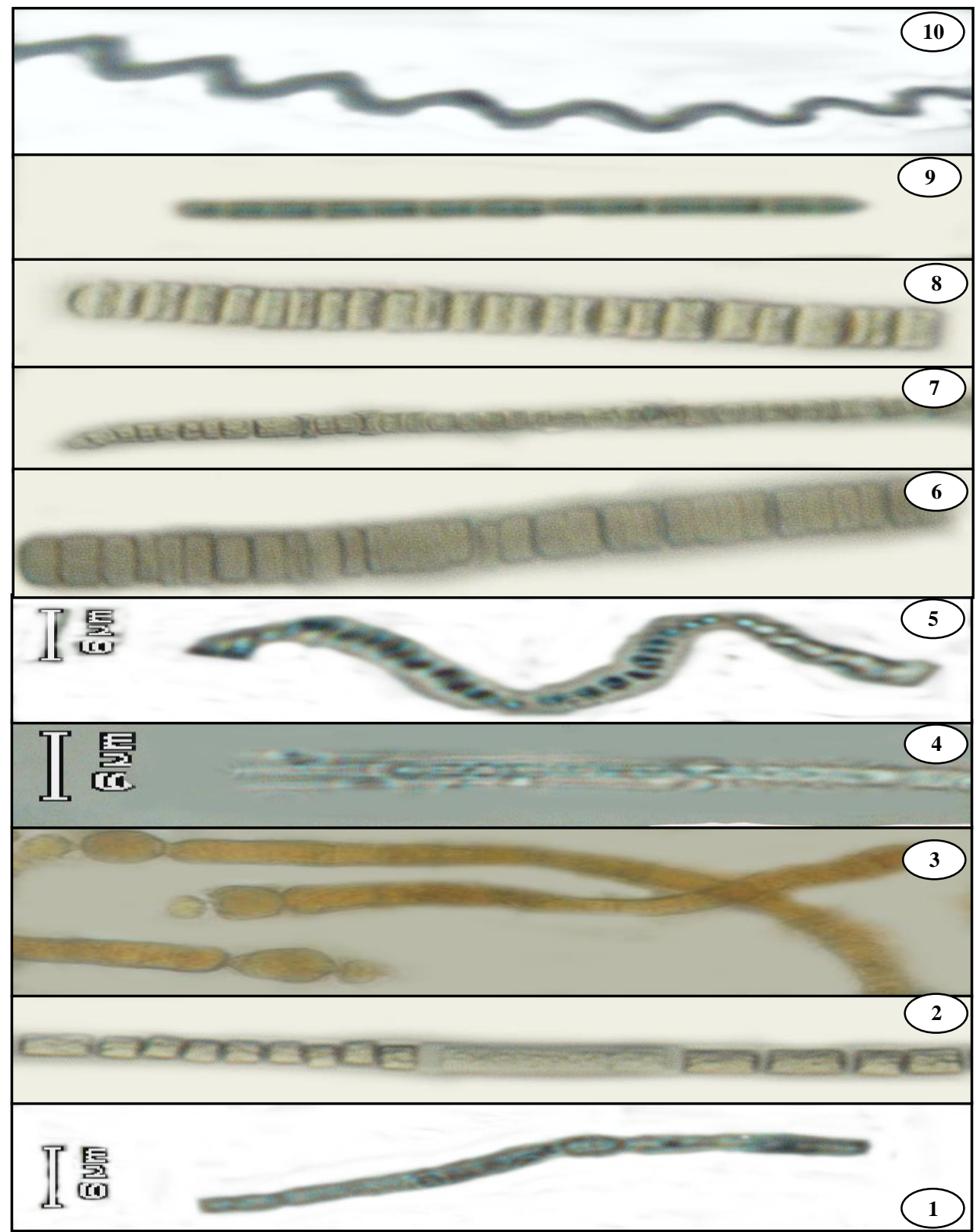


and Calonies silicula) were found on Ceratophyllum demersum. At site (I) on Myriophyllum spicatum, diatom species (Nitzschia sigmoidea, Gomphonema olivaceum, Synedra capitata, Calonies amphisbaena, Gomphonema truncatum, Stauronies anceps, Amphora ovalis, Cymbella affinies, Cymbella tumida and Cyclotella operoulata) were found.

The lowest numbers of diatom species were found on Echhornia crassipes in site (I and II) and (IV). Synedra acus var. angustissima and Amphora ovalis were found in site (I) but Cymbell cistula was found in site (IV). Four species of Oedogonium were found only in site (II) and one species of Cyanophyta (Spirulina laxissima) was found in site (I).

Elsewhere, plant species and their architecture and of plant density have strong effects on the development of epiphytic organisms (Cattaneo and Kalf, 1980; Cattaneo et al., 1988). Rychkova (1989) stated that, epiphytic algal biomass is largely eliminated by changes in the water action, which mainly detach the loosely attached species. In summer Chlorococcales and filamentous Chlorophytes, including some planktonic and periphytic species, such as (Scenedesmus spp., Pediastrum spp., Oedogonium spp. and Spirogyra spp.) and some Cyanophytes such as Oscillatoria spp., Anabaena spp. and Spriulina spp. reached considerable numbers. As Bouvy et al. (1997) pointed out that periphyton and phytoplankton may exchange organisms and compete for nutrient.

\section{References}

Albay, M. and Aykulu, G. (2002). Invertebrate grazer-epiphytic algae interaction on submerged macrophytes in a mesotrophyic Turkish lake. E.U. Journal of Fisheries and Aquatic Sciences, 19(1-2): 247-258.

American public health association (APHA). (1981). Standard methods for the examination of water and waste water, 15 ed., New York, 1134 pp

Bourrelly, P. (1968). Les algues d'eau douce initiation a la systematique. Tome11. Les Algues jaunes et brunes, Chrysophycées, Xanthophycées et Diatomées Ed. N. Boubee and Cie, Paris, France, pp.438.

Bourrelly, P. (1970). Les algues d'eau douce initiation a la systematique. Tome111. Les Algues bleus et rouges. Ed. N. Boubee and Cie, Paris, France, pp.512.

Bouvy, M.; Arfi, R. and Troussellier, M. (1977). Taxonomic characterization of pelagic and periphytic heterotrophic bacteria isolated from the tropical Ebrie Lagoon Cote d'Ivoire. Arch.Hydrobiol., 140:3:393-409.

Burkholder, J. M.; Wetzel, R. G and Komparens, K.L. (1990). Direct comparison of phosphate by adnate and loosely attached micro-algae within an intact biofilm matrix. Appl. Environ. Microbiol., 56:28822990.

Burkholder, J. M. and Wetzel, R.G. (1990). Epiphytic alkaline Phosphatase on natural and artificial plants in an oligotrophic lake: re-evaluations of the 
role of macrophytes as a phosphorus source for epiphytes. Limnol. Oceanogr., 35:736-747.

Cattaneo, A. and Kalff, J. (1980). The relative contribution of aquatic macrophytes and their epiphytes to the production of macrophytes bed. Limnol. Oceanogr., 25:280-289.

Cattaneo, A.; Galanto, G.; Gentinetta, S. and Roma, S. (1988). Epiphytic algae and macro-invertebrates on submerged and floating-leaved macrophytes in an Italian lake. Fresh water Biology, 39:725-740.

Cattaneo, A. and Kalff, J. (1978). Seasonal changes in the epiphyte community of natural and artificial macrophytes in Lake Memphremagog (Que And Vt.). Hydrobiologia, 60: 135-144.

Corlett, H. and Jones, B. (2007). Epiphytic communities on Thalassia testudinum from Grand Cayman, British, West Indies: Their composition, structure, and contribution to lagoonal sediments. Sedimentary Geology, 194:245-262.

Cox, E.J. (1996). Identification of fresh water diatoms from live materialChapman and Hall, 2-6 Boundary Row, London SE .18, HN, UK., 156 p.

Dere, S.; Karacaoglu, D. and Dalkiran, N.( 2002):A study on the Epiphytic algae on the Nilufer stream (Bursa),Turk.J.Bot.,26:219-233.

Deutsche Einheitsverfahren Zur Wasser, Abwasser, und SchlammUntersuchung [German standard procedures for testing water, wastewater and sludge.] (1960). Verlag Chemie, Weinheim

Dewis, J. and Freitas, F. (1970). In physical and chemical methods of soil and water analysis, Soil Bulletin, No. 10. food and the occurrence of bluegreen algae in peat soils. British Phycological Journal, 8: 289-293.

El-Shahed, A. M. and Fathy, A. A. (2000). Diatom assemblages associating Cladophora glomerata (L.) Kutz in Egyptian fluvial environments. Bull. Fac. Sci., Assiut Univ., 29 (I.D):357- 366.

Goldsborough, L. G. and Hickman, M. (1991). A comparison of epiphytic algal biomass and community structure on Scripus validus and on a morphologically similar artificial substratum. J. Phycol., 27:916-207.

Jackson, M. L. (1977). Soil chemical analysis. Prentice-Hall of India, private limited New Delhi, 498 pp.

Kitting, C. L.; Fry, B. and Morgan, M. D. (1984). Detection of inconspicuous epiphytic algae supporting food webs in sea grass meadows. Ocologia (Berlin), 2:145-149.

Muller, U. (1996). Production rates of epiphytic algae in an eutrophic lake. Hydrobiolog., 330: 37-45.

Obali, O.; Gönülol, A. and Dere, Ş. (1989). Algal flora in the littoral zone of Lake Mogan. 19 Mayis Üniv Fen Derg, 3: 33-53.

Prescott, G.W. (1978). How to know the fresh water algae. Brown Company publishers Dubuque, Iowa, USA, pp 12.

Egyptian J. of Phycol. Vol. 11, $2010 \quad$ - 118 - 
Rychkova, M.A. (1989). Role of water mass dynamics information of epiphytic algae communities in a lake. Hydrobiological Journal, 25(3):157-191.

Sand-Jensen, K.; Borg, D. and Jeppesen, E. (1989). Biomass and oxygen dynamics of the epiphyte community in a Danish lowland stream. Freshwater Biol., 22:431-443.

Saunders, J.; Attrill, M.J.; Shaw, S. M. and Rowden, A. A. (2003). Spatial variability in the epiphytic algal assemblages of Zostera martina sea grass beds. Mar. Ecol. Prog. Ser., 249:107-115.

Schwarzenbach, G. and Biedermann, W. (1948). Komplexone, X. Erdalkalikomplexe vono. 0, 6-Dioxyazofarbstoffen. Helv. Chim. Acta, 31: 678-687.

Şen, B. and Aksakal, M. (1988). The Seasonal Changes and density of epiphytic algae population on Potamogeton sp. and Nasturtium officinal in Kirkgozeler (in turkish). IX. National Biology Congress, 21-23 September Vol. 3; Sivas, Turkey.

Strickland, J. D. H. and Parasons, T. R. (1965). A manual of sea water analysis $\left(2^{\text {nd }}\right.$ ed.), Bull. No. (125) of the fish Res. Board of Canada; Ottawa, 203 pp.

Sullivan, M .J. and Moncrreif, C. A. (1990). Edaphic algae are an important component of salt march food webs evidence from multiple stable isotope analyses. Mar. Ecol. Prog. Ser., 62:149-159.

Wetzel, R .G. (1983). Periphyton in Ecosystems. Dr. W. Junk. Boston, 346 pp.

Woods, J. T. and Mellon M. A. (1941). Chlorostannous- molybdophosphoric blue colour method, in sulfuric acid system. In Soil Chemical Analysis by Jackson, M.L. (1958): Printice Hall Iinternational, Inc., London.

Yilditz, k. (1987). A study on algal assemblage of Altmapa Dam Lake and its outlet Cumhuriyet University, Journal of Science, 5:191-207.

Zimba, P.V. (1995). Epiphytic biomass in the littoral zone, Lake Okeechobee, Florida (USA). Arch. Hydrobiol. Spec. Issues Advanc. Limnol., 45:233240.

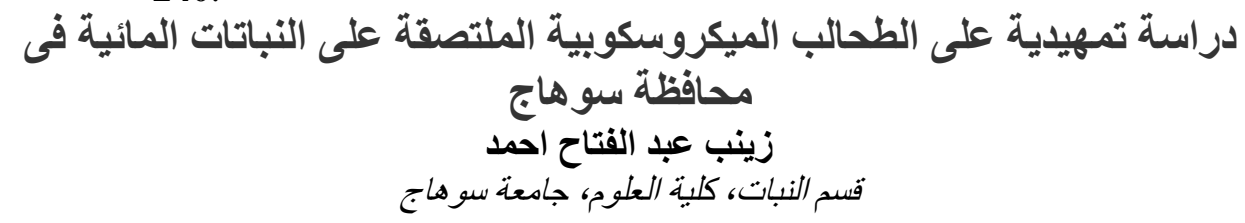

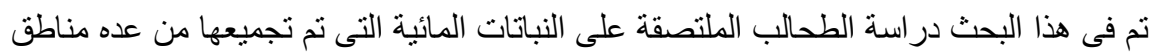

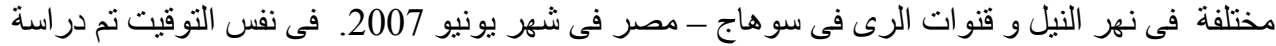

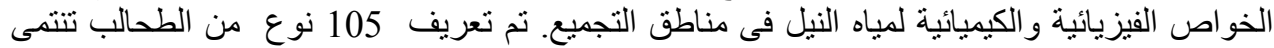

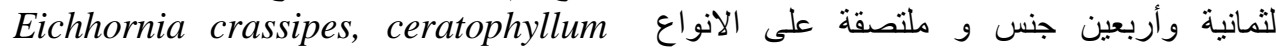
demersum, Myriophyllum spicatum, phragmites australis)

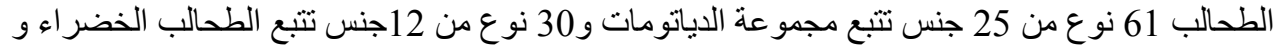

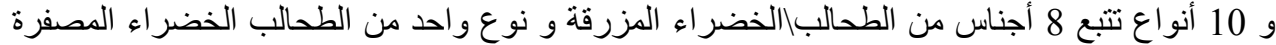
وثثلاث أنو اع من ثلاث اجناس تتبع الطحالب اليوجلينية. 